\title{
Inhibition of lateral shoot formation by RNA interference and chemically induced mutations to genes expressed in the axillary meristem of Nicotiana tabacum L.
}

\author{
Kaori Hamano* ${ }^{*}$, Seiki Sato, Masao Arai, Yuta Negishi, Takashi Nakamura, Tomoyuki Komatsu,
} Tsuyoshi Naragino and Shoichi Suzuki

\begin{abstract}
Background: Lateral branches vigorously proliferate in tobacco after the topping of the inflorescence portions of stems for the maturation of the leaves to be harvested. Therefore, tobacco varieties with inhibited lateral shoot formation are highly desired by tobacco farmers.

Results: Genetic inhibition of lateral shoot formation was attempted in tobacco. Two groups of genes were examined by RNA interference. The first group comprised homologs of the genes mediating lateral shoot formation in other plants, whereas the second group included genes highly expressed in axillary bud primordial stages. Although "primary" lateral shoots that grew after the plants were topped off when flower buds emerged were unaffected, the growth of "secondary" lateral shoots, which were detected on the abaxial side of the primary lateral shoot base, was significantly suppressed in the knock-down lines of NtLs, NtB/1, NtREV, VE7, and VE12. Chemically induced mutations to NtLs, NtB/1, and NtREV similarly inhibited the development of secondary and "tertiary" lateral shoots, but not primary lateral shoots. The mutations to NtLs and NtB/1 were incorporated into an elite variety by backcrossing. The agronomic characteristics of the backcross lines were examined in field trials conducted in commercial tobacco production regions. The lines were generally suitable for tobacco leaf production and may be useful as new tobacco varieties.

Conclusion: The suppressed expression of NtLs, NtB/1, NtREV, VE7, or VE12 inhibited the development of only the secondary and tertiary lateral shoots in tobacco. The mutant lines may benefit tobacco farmers by minimizing the work required to remove secondary and tertiary lateral shoots that emerge when farmers are harvesting leaves, which is a labor-intensive process.
\end{abstract}

Keywords: Chemically induced mutation, Lateral shoot inhibition, RNAi, RNA-seq, Sucker, Tobacco

\section{Background}

Lateral shoot development, which is a fundamental process in plants, is uniquely regulated in all species. In crop species, harvest quality and productivity are directly affected by

*Correspondence: kaori.hamano@jt.com

Leaf Tobacco Research Center, Japan Tobacco Inc., 1900 Idei, Oyama, Tochigi 323-0808, Japan how lateral shoot formation is controlled. Genes involved in axillary meristem development have been studied in various plant species. Mutations in Lateral suppressor genes in tomato (Ls) [1] and Arabidopsis (LAS) [2] inhibit axillary shoot formation during the vegetative phase. A mutation in a rice ortholog, Monoculm 1 (MOC1), results in suppressed tillering and a decrease in the number of rachis branches and spikelets [3]. A mutation in the Blind gene strongly suppresses 
axillary meristem formation in tomato $[4,5]$. Three Regulator of Axillary Meristems (RAX) genes, RAX1, RAX2, and $R A X 3$, were identified in Arabidopsis as homologs of Blind. Triple recessive mutants exhibit almost completely inhibited axillary shoot formation [6, 7]. Blind and $R A X$ genes encode R2R3 MYB transcription factors, which are critical for initiating axillary meristem development during the vegetative phase. Previous studies revealed that Blind also controls axillary meristem initiation in the reproductive phase $[5,6]$. The decapitation of primary shoots does not stimulate the outgrowth of axillary shoots in the Arabidopsis las mutant or $\operatorname{rax} 1$ mutant $[2,7]$. The $L A X 1$ gene in rice and the $B A 1$ gene in maize encode bHLH domains and are involved in the branching of inflorescence and vegetative shoots $[8,9]$. The $R O X$ gene in Arabidopsis is an ortholog of $L A X 1$ and $B A 1$. It is reportedly involved in lateral shoot formation in the early vegetative stage [10].

Mutations in some genes regulating axillary meristem formation affect the development of the main shoot apices. For example, a mutation in the Revoluta (REV) gene in Arabidopsis substantially decreases the outgrowth of rosette and cauline leaves and inhibits the stimulation of axillary shoots following the decapitation of primary shoots [11, 12]. Additionally, the rev mutation sometimes leads to the arrested development of the primary shoot apical meristem (SAM) at an early stage. The Cup-Shaped Cotyledon (CUC) genes CUC1, CUC2, and CUC3 [1315] and the Lateral Organ Fusion (LOF) gene [16] regulate organ separation and axillary meristem formation in Arabidopsis. Hairy Meristem (HAM) genes in petunia [17] and pepper [18] and their homolog in Arabidopsis, Lost Meristem (LOM) [19], are important for SAM and axillary meristem development. Consequently, petunia and pepper ham mutants and the Arabidopsis lom1lom2-lom3 triple mutant exhibit premature termination of the shoot apex and arrested axillary shoot development. A mutation in the Far-Red Elongated Hypocotyl $(F H Y 3)$ gene in Arabidopsis leads to inhibited axillary bud outgrowth. Additionally, the rev-fhy3 double mutation represses axillary bud formation considerably more than the single recessive mutations [20].

Because tobacco is cultivated for its leaves, when plants start to flower, the apical portions of stems (mainly inflorescences) are cut off (i.e., topping) to enhance leaf development and maturation prior to harvest. Although tobacco plants generally exhibit strong apical dominance, topping releases lateral buds from dormancy. The resulting lateral shoots, which are often called "suckers" in tobacco, undergo vigorous development. Tobacco farmers must remove these "primary" lateral shoots soon after they develop, but "secondary" lateral shoots grow immediately from the abaxial side of the primary lateral shoot base. Thus, lateral shoots emerge sequentially during the cultivation period (up to "tertiary" lateral shoots). These lateral shoots must be removed manually or suppressed by chemicals (i.e., "suckercides") to enable plants to produce many high-quality leaves. However, this control of lateral shoots is a labor-intensive and costly process. Removing the secondary and tertiary lateral shoots is especially inconvenient because the timing of their emergence coincides with when farmers must harvest the leaves, which is another labor-intensive task. Therefore, tobacco varieties with fewer lateral shoots, especially secondary and tertiary ones, are highly desired. Unfortunately, breeding involving conventional and biotechnological approaches has not resulted in varieties with substantially fewer lateral shoots.

The tobacco genes involved in the regulation of axillary shoot development and their functions remain relatively uncharacterized. Nevertheless, it is possible that homologs of the above-mentioned genes in other plants are present and function similarly in tobacco. For example, a tobacco homolog (GenBank EU935981) of Ls has been cloned [21], but its function was not investigated. In this study, the expression of the homologs of these genes as well as a number of other genes preferentially expressed in the axillary meristems was knocked down in tobacco by RNA interference (RNAi). Additionally, the chemically induced mutations to selected genes inhibited lateral shoot formation. Mutant lines were characterized in greenhouse and field trials.

\section{Results \\ BLAST search}

Two groups of tobacco genes were examined to assess the effects of RNAi knock-down on lateral shoot formation. The first group included homologs of the genes involved in lateral shoot formation in other plants. A BLAST search of the GenBank database and an in-house tobacco cDNA database was conducted to identify tobacco homologs (Table 1). Because tobacco is an amphidiploid species that inherited its genome from Nicotiana sylvestris (S-genome) and Nicotiana tomentosiformis (T-genome) [22-24], both $\mathrm{S}$-genes and $\mathrm{T}$-genes were identified for each homolog. The $N t B l$ and $N t C U C$ genes were numbered respectively in descending order of homology with Blind and Goblet (GenBank HM210879), a tomato homolog of the $C U C$ genes.

\section{RNA sequencing (RNA-seq) analysis}

Genes that were not studied in other plants might also be involved in the regulation or development of axillary meristems and shoots. Thus, as the second group, genes that are highly expressed in axillary bud primordial stages were identified by RNA-seq. Tissues were sampled from axillary meristem zones in the early stage (EA) and 
Table 1 Tobacco homologs of genes involved in the regulation or development of axillary meristems and shoots in other species

\begin{tabular}{|c|c|c|c|}
\hline \multirow[t]{2}{*}{ Query gene (Species, GenBank accession) for BLAST search } & \multirow[t]{2}{*}{$\begin{array}{l}\text { Tobacco } \\
\text { homologue }\end{array}$} & \multicolumn{2}{|c|}{$\begin{array}{l}\text { Accession number in GenBank or Sol Genomics } \\
\text { Network }^{\mathrm{a}}\end{array}$} \\
\hline & & S-gene & T-gene \\
\hline Ls (Tomato, AF098674) & NtLs & EU935581 & AM848584 \\
\hline \multirow[t]{3}{*}{ MYB domain of Blind (Tomato, AF426174.1) } & $N+B / 1$ & Nitab4.5_0001442g0010.1 & Nitab4.5_0004993g0020.1 \\
\hline & $N+B / 2$ & Nitab4.5_0001050g0010.1 & Nitab4.5_0001163g0150.1 \\
\hline & $N+B / 3$ & Nitab4.5_0007679g0010.1 & Nitab4.5_0000578g0120.1 \\
\hline REV (Tomato, BT013577 and Arabidopsis, AF233592) & NtREV & JQ686937 & Nitab4.5_0004624g0050.1 \\
\hline \multirow{4}{*}{$\begin{array}{l}\text { NAC domains of CUC genes (Arabidopsis, NM_112380, NM_124774, } \\
\text { and NM_106292) }\end{array}$} & NtCUC1 & Nitab4.5_0007278g0010.1 & Nitab4.5_0000568g0080.1 \\
\hline & NtCUC2 & Nitab4.5_0008840g0010.1 & Nitab4.5_0005914g0020.1 \\
\hline & NtCUC3 & Nitab4.5_0004286g0020.1 & Nitab4.5_0007189g0080.1 \\
\hline & NtCUC4 & XM_016587094 & XM_016602286.1 \\
\hline FHY3 (Arabidopsis, NM_001125201) & $\mathrm{NtFHY3}$ & Nitab4.5_0002232g0050.1 & XM_016593996.1 \\
\hline LOM (Arabidopsis, NM_130079.3) & NtLOM1 & Nitab4.5_0011278g0010.1 & XM_016625781.1 \\
\hline LOF (Arabidopsis, NM_001160897.2) & NtLOF1 & XM_016601029.1 & XM_016602279.1 \\
\hline
\end{tabular}

The tobacco $B$ l and CUC homolog number was determined on the basis of the sequence similarity to a tomato B/ gene (AF426174.1) and CUC gene (HM210879.1), respectively

${ }^{\text {a }}$ Homologs originally identified in the in-house database were later submitted to the Sol Genomics Network database (https://solgenomics.net/) and GenBnak (https://www.ncbi.nlm.nih.gov/genbank/) by other research groups. Therefore, the accession numbers in the public database are provided

the very early stage (VE) and from the control zones of tobacco plants by laser micro-dissection (Fig. 1). Total RNA was extracted from the tissues and analyzed using the 454 GS FLX next-generation sequencer (454 Life Sciences, Roche). Approximately 900,000 reads, with an average length of approximately $400 \mathrm{bp}$, were obtained for each tissue sample and were assembled into 38,569 unique genes. The second group of genes was selected according to the following criteria: (a) the read count of a gene in EA or VE was at least 10-times higher than that in the control, (b) the assembled gene was longer than $200 \mathrm{bp}$, and (c) the gene encodes a transcription factor or unknown protein according to the annotation. We selected 11 genes highly expressed in EA tissue (EA1-EA11) and 13 genes highly expressed in VE tissue (VE1-VE13) (Table 2).

The RNA read counts for the first group of genes (i.e., tobacco homologs) are listed in Table 2. A comparison with the control tissue indicated NtLs and NtBl1 were highly and specifically expressed in EA or VE tissue. In all three samples, NtREV was highly expressed. Regarding

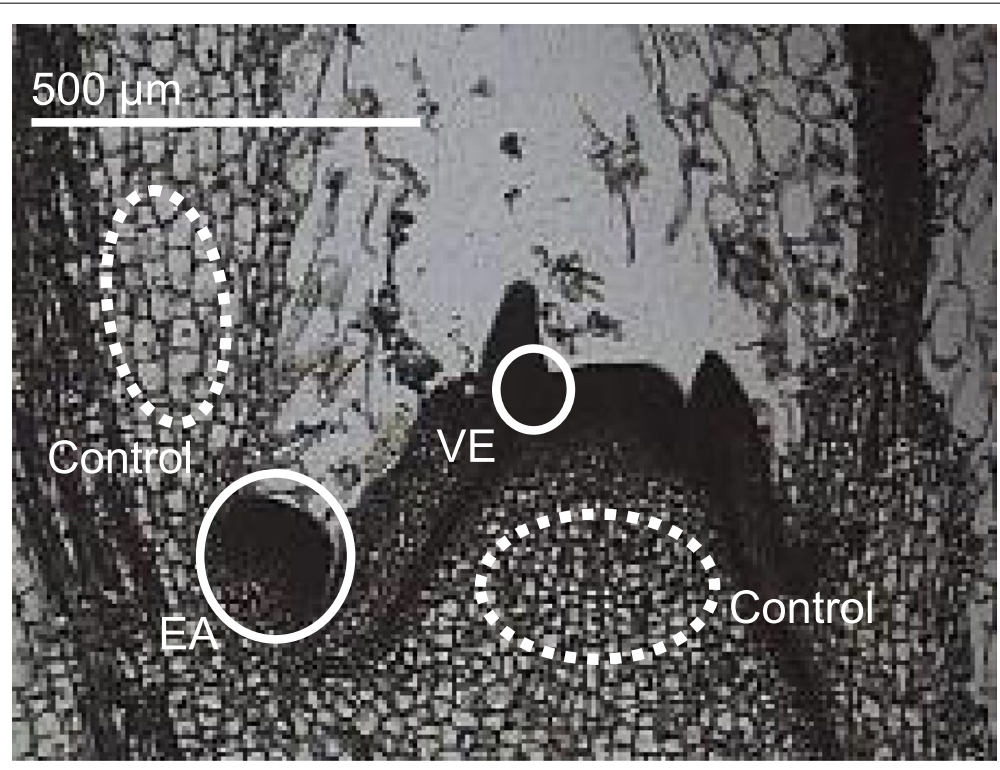

Fig. 1 Tissues at early (EA) and very early (VE) axillary meristem developmental stages and control tissues sampled by laser micro-dissection 
the other homologs, there were no significant differences in the expression levels among the three tissues or the expression levels were too low to detect. Earlier investigations proved that $L A S$ and $R A X$ genes are highly expressed in the axil of leaf primordia [2, 6, 7]. Additionally, $R E V$ is reportedly expressed in the initial lateral shoot meristem as well as in the center of the SAM in an inverted-cup-shaped cell population [12]. Consequently, the expression patterns of at least some of the homologs
(NtLs, NtBl1, and NtREV) were similar to those of their counterparts in other species.

Notably, the sequence analysis revealed that VE7, which encodes a bHLH domain, is a homolog of the $R O X$ gene in Arabidopsis. The identification of the $R O X$ was published after our BLAST search for homologs of the genes described above [10]. Additionally, VE12, which encodes a NAC domain and lacks a recognition site for miR164, was identified as a CUC3 homolog.

Table 2 Expression of the genes in the early axillary meristem tissues and effects of RNA interference

\begin{tabular}{|c|c|c|c|c|c|c|c|c|}
\hline \multirow[t]{3}{*}{ Gene } & \multirow{2}{*}{\multicolumn{3}{|c|}{$\begin{array}{l}\text { RNAseq counts (Reads Per Kilobase of } \\
\text { exon per Million mapped sequence } \\
\text { reads) }\end{array}$}} & \multicolumn{5}{|c|}{ Effect of RNAi measured relative to null segregants \pm SD } \\
\hline & & & & \multirow[t]{2}{*}{ RNA expression } & \multicolumn{2}{|c|}{ Primary lateral shoots } & \multicolumn{2}{|c|}{ Secondary lateral shoots } \\
\hline & EA & VE & Control & & Number & Weight & Number & Weight \\
\hline NtLs & 1.16 & 62.97 & 1.14 & $0.50 \pm 0.08$ & $1.00 \pm 0.00$ & $1.28 \pm 0.15$ & $0.13 \pm 0.09^{\mathrm{a}}$ & $0.1 \pm 0.06^{\mathrm{a}}$ \\
\hline NtB/1 & 12.80 & 108.85 & 8.43 & $0.31 \pm 0.05$ & $1.02 \pm 0.06$ & $1.21 \pm 0.18$ & $0.00 \pm 0.00^{\mathrm{a}}$ & $0.00 \pm 0.00^{\mathrm{a}}$ \\
\hline$N+B / 2$ & ND & ND & ND & $0.19 \pm 0.04$ & $1.00 \pm 0.00$ & $1.13 \pm 0.15$ & $1.00 \pm 0.10$ & $1.46 \pm 1.65$ \\
\hline$N+B / 3$ & 11.88 & 23.20 & 10.26 & $0.30 \pm 0.09$ & $1.00 \pm 0.00$ & $1.45 \pm 0.48$ & $0.88 \pm 0.46$ & $0.77 \pm 0.12$ \\
\hline NtREV & 110.74 & 167.99 & 305.61 & $0.62 \pm 0.10$ & $1.00 \pm 0.00$ & $0.98 \pm 0.09$ & $0.06 \pm 0.10^{\mathrm{a}}$ & $0.03 \pm 0.04^{\mathrm{a}}$ \\
\hline NtCUC1 & 4.24 & 10.32 & 8.38 & $0.16 \pm 0.07$ & $1.00 \pm 0.00$ & $1.05 \pm 0.12$ & $0.91 \pm 0.18$ & $0.57 \pm 0.10$ \\
\hline NtCUC2 & ND & $N D$ & ND & $0.53 \pm 0.04$ & $1.00 \pm 0.00$ & $1,14 \pm 0.26$ & $0.97 \pm 0.12$ & $1.06 \pm 0.4$ \\
\hline NtCUC3 & ND & ND & ND & $0.17 \pm 0.10$ & $1.00 \pm 0.00$ & $0.96 \pm 0.17$ & $1.08 \pm 0.01$ & $0.71 \pm 0.26$ \\
\hline NtCUC4 & ND & ND & ND & $0.69 \pm 0.03$ & $1.00 \pm 0.00$ & $0.91 \pm 0.04$ & $1.05 \pm 0.09$ & $0.75 \pm 0.19$ \\
\hline $\mathrm{NtFHY}_{3}$ & 23.49 & 36.90 & 46.59 & $0.31 \pm 0.17$ & $1.00 \pm 0.00$ & $0.94 \pm 0.08$ & $1.03 \pm 0.11$ & $1.12 \pm 0.62$ \\
\hline NtLOM1 & 36.69 & 49.15 & 65.87 & $0.38 \pm 0.06$ & $1.00 \pm 0.00$ & $1.09 \pm 0.15$ & $1.72 \pm 1.97$ & $2.56 \pm 3.99$ \\
\hline NtLOF1 & 30.27 & 60.57 & 25.56 & $0.38 \pm 0.11$ & $1.01 \pm 0.02$ & $1.67 \pm 0.15$ & $0.28 \pm 0.30$ & $0.64 \pm 0.58$ \\
\hline EA1 & 32.93 & 2.30 & $N D$ & $0.09 \pm 0.03$ & $1.00 \pm 0.00$ & $1.14 \pm 0.16$ & $0.97 \pm 0.15$ & $1.21 \pm 0.34$ \\
\hline EA2 & 20.13 & 9.70 & 1.48 & $0.28 \pm 0.16$ & $1.00 \pm 0.00$ & $1.17 \pm 0.35$ & $1.34 \pm 0.82$ & $1.65 \pm 1.59$ \\
\hline$E A 3$ & 22.56 & 1.77 & ND & $0.16 \pm 0.03$ & $1.00 \pm 0.00$ & $0.93 \pm 0.09$ & $0.68 \pm 0.16$ & $1.42 \pm 1.53$ \\
\hline EA4 & 43.22 & 2.50 & 1.19 & $0.35 \pm 0.17$ & $1.00 \pm 0.00$ & $1.16 \pm 0.28$ & $1.14 \pm 0.41$ & $1.09 \pm 0.38$ \\
\hline EA5 & 14.41 & ND & ND & $0.10 \pm 0.04$ & $1.00 \pm 0.00$ & $1.04 \pm 0.07$ & $4.05 \pm 2.21$ & $18.88 \pm 28.34$ \\
\hline EA6 & 22.78 & ND & ND & $0.80 \pm 0.22$ & $1.00 \pm 0.00$ & $1.01 \pm 0.26$ & $1.04 \pm 0.27$ & $1.30 \pm 0.80$ \\
\hline EA7 & 12.14 & ND & ND & $0.10 \pm 0.01$ & $1.00 \pm 0.00$ & $1.02 \pm 0.17$ & $0.83 \pm 0.76$ & $0.77 \pm 0.70$ \\
\hline$E A 8$ & 43.57 & ND & 1.47 & $0.02 \pm 0.02$ & $1.00 \pm 0.00$ & $1.08 \pm 0.36$ & $1.14 \pm 0.11$ & $1.21 \pm 0.2$ \\
\hline EA9 & 14.03 & ND & ND & $0.17 \pm 0.12$ & $1.00 \pm 0.00$ & $0.95 \pm 0.17$ & $1.10 \pm 0.77$ & $1.16 \pm 0.74$ \\
\hline EA10 & 51.93 & 8.72 & 1.25 & $0.43 \pm 0.08$ & $1.00 \pm 0.00$ & $1.33 \pm 0.16$ & $1.59 \pm 0.99$ & $0.96 \pm 0.21$ \\
\hline EA11 & 19.63 & 1.70 & 1.62 & $0.56 \pm 0.08$ & $1.00 \pm 0.00$ & $0.98 \pm 0.27$ & $0.98 \pm 0.09$ & $1.52 \pm 0.92$ \\
\hline VE1 & 39.71 & 49.37 & 3.79 & $0.58 \pm 0.06$ & $1.00 \pm 0.00$ & $0.99 \pm 0.30$ & $1.10 \pm 0.16$ & $1.27 \pm 0.51$ \\
\hline VE2 & 26.70 & 32.21 & 0.69 & $0.51 \pm 0.02$ & $1.00 \pm 0.00$ & $1.04 \pm 0.12$ & $1.09 \pm 0.17$ & $0.95 \pm 0.54$ \\
\hline VE3 & 6.51 & 49.14 & ND & $1.51 \pm 0.05$ & $1.00 \pm 0.00$ & $1.16 \pm 0.21$ & $1.02 \pm 0.45$ & $0.97 \pm 0.35$ \\
\hline VE4 & ND & 10.94 & ND & $0.32 \pm 0.04$ & $1.00 \pm 0.00$ & $1.27 \pm 0.31$ & $0.80 \pm 0.38$ & $0.95 \pm 0.64$ \\
\hline VE5 & 20.88 & 79.27 & 3.12 & $0.41 \pm 0.06$ & $1.00 \pm 0.00$ & $1.00 \pm 0.17$ & $1.3 \pm 0.74$ & $1.41 \pm 0.84$ \\
\hline VE6 & 2.61 & 31.26 & ND & $0.35 \pm 0.14$ & $1.00 \pm 0.00$ & $0.73 \pm 0.12$ & $2.06 \pm 0.56$ & $4.57 \pm 3.20$ \\
\hline VET & ND & 73.71 & 4.97 & $0.08 \pm 0.02$ & $1.00 \pm 0.00$ & $1.49 \pm 0.30$ & $0.33 \pm 0.09^{a}$ & $0.26 \pm 0.11^{\mathrm{a}}$ \\
\hline VE8 & 5.93 & 13.38 & ND & $0.14 \pm 0.06$ & $1.00 \pm 0.00$ & $1.01 \pm 0.28$ & $1.41 \pm 0.43$ & $0.87 \pm 0.39$ \\
\hline VE9 & 5.06 & 14.07 & ND & $0.62 \pm 0.08$ & $1.00 \pm 0.00$ & $0.98 \pm 0.10$ & $1.03 \pm 0.16$ & $0.90 \pm 0.12$ \\
\hline VE10 & 9.42 & 21.58 & 1.86 & $0.42 \pm 0.04$ & $1.00 \pm 0.00$ & $0.97 \pm 0.25$ & $1.16 \pm 0.62$ & $1.79 \pm 1.29$ \\
\hline VE11 & ND & 13.62 & ND & $0.70 \pm 0.07$ & $1.00 \pm 0.00$ & $1.07 \pm 0.28$ & $0.58 \pm 0.56$ & $0.42 \pm 0.43$ \\
\hline VE12 & 19.79 & 133.95 & 11.71 & $0.18 \pm 0.06$ & $1.00 \pm 0.00$ & $1.22 \pm 0.12$ & $0.29 \pm 0.41$ & $0.05 \pm 0.08^{\mathrm{a}}$ \\
\hline VE13 & 5.68 & 19.24 & ND & $0.14 \pm 0.05$ & $1.00 \pm 0.00$ & $1.03 \pm 0.21$ & $1.03 \pm 0.12$ & $1.86 \pm 0.94$ \\
\hline
\end{tabular}

ND Not detectable

${ }^{a}$ Significantly different from null segregants at the $1 \%$ level as determined by the $t$-test, assuming that the means of the relative values were from normal distributions 
Previous studies confirmed that $R O X[10]$ and its maize ortholog BA1 [9] are expressed at the adaxial boundary of leaf primordia and that $C U C 3$ is expressed at the boundaries between leaf primordia and the shoot meristem [25]. Accordingly, VE7 and VE12 are similar to the homologs regarding their expression patterns. In addition to the early stage of axillary meristem, $L s, B l 1$, and most of the $V E$ and $E A$ genes were expressed in other tissues and organs (Supplemental Table S1). More importantly, the RNA-seq analysis supplemented the search for homologs.

\section{RNAi knock-down}

A total of 36 trigger sequences, with an average length of $430 \mathrm{bp}$, were designed for the RNA interference of the 12 homologs and 24 genes selected during the RNA-seq screening (Supplemental Table S2). Three $\mathrm{NtBl}$ and four $N t C U C$ genes were obtained. Therefore, the less conserved regions (i.e., less than $70 \%$ identity) of the three $\mathrm{NtBl}$ genes and the four $\mathrm{NtCUC}$ genes were used for designing trigger sequences to distinguish each gene. For each target, the S-gene and T-gene were compared. A highly homologous region was chosen as the trigger to ensure that both genes were effectively knocked down by a single trigger sequence. The average sequence identity between the trigger regions of the $\mathrm{S}$-genes and $\mathrm{T}$-genes was $95.4 \%$, which was higher than the homology sufficient for simultaneously knocking down two genes determined by Parrish et al. [26].

The RNAi genes were introduced into Nicotiana tabacum L. cv. Petit Havana SR-1. The $\mathrm{T}_{1}$ progeny of three single-locus transformants for each construct were grown in a greenhouse and were examined regarding target gene expression. The target genes were thoroughly suppressed in the analyzed plants (Table 2), with the exception of the VE3 transformants, in which the target gene was more highly expressed than in the null segregants; the reasons for the increased expression remain unknown. The data revealed a lack of difference in primary lateral shoot production (i.e., number and weight) between the transgenic plants and the null segregants (Table 2). Therefore, the knock-down of target gene expression by RNAi did not suppress primary lateral shoot formation. However, the production of secondary lateral shoots was suppressed in terms of number and/or weight in the transformants in which $N t L s$, $N t B l 1, N t R E V, V E 7$, and VE12 were targeted. For example, a secondary lateral bud was visible at the base of the abaxial side of a primary lateral shoot in wild-type plants (Fig. 2a), but not in transgenic plants in which $N t L s$ expression was knocked down (Fig. 2b). After the secondary lateral shoots were removed, no additional lateral shoots were formed on plants grown in the greenhouse.
An earlier investigation indicated that a sequence match of at least 22 consecutive nucleotides may result in offtarget silencing by a trigger sequence [27]. Therefore, we performed a BLAST search to screen for 22 consecutive nucleotides identical to the trigger sequences designed for the five effective genes (i.e., NtLs, NtBl1, NtREV, VE7, and $V E 12)$. With the exception of $V E 12$, there were no tobacco genes with 22 consecutive nucleotides identical to the trigger sequences. The $V E 12$ gene is considered to be a NAC gene, which belongs to one of the largest transcription factor families in tobacco [28]. Therefore, we cannot exclude the possibility of off-target silencing in the VE12_RNAi tobacco plants. Regarding NtBl1, we confirmed that the expressions of $N t B l 2$ and $N t B l 3$ were not down-regulated in the NtBl1_RNAi tobacco plants. Therefore, the RNAi results were validated further.

\section{Chemically induced mutants}

The ethyl methanesulfonate (EMS) mutant library for Nicotiana tabacum L. cv. Tsukuba 1 was screened for nonsense mutations in NtLs, NtBl1, NtREV, VE7, and $V E 12$. The following mutations were identified and mapped (Fig. 3): two each in NtLs-S and NtBl1-S and one each in NtLs-T, NtBl1-T, NtRev-S, and NtRev-T. Moreover, mutations in the S-genome and T-genome were combined by crossing the mutants, producing double-mutant lines designated as SAS lines (Table 3). Because nonsense mutations were not identified in VE7 and VE12, these genes were not studied further.

Mutations in some genes involved in axillary meristem formation reportedly affect the development of the main shoot apices [11-19]. The seedlings of the SAS-rev mutant lines produced cup-shaped or asymmetric cotyledons (Fig. 2g). Additionally, the leaves of 4- to 5-week-old Coker319-ls-1 plants, backcross line for field trials at commercial production sites, grown in nursery boxes occasionally turned yellow (Fig. 2i). The same phenotype was observed in 4- to 5-week-old Coker319- $l s-1$ plants incubated at $15{ }^{\circ} \mathrm{C}$ in a growth chamber, although the plants appeared normal at $28{ }^{\circ} \mathrm{C}$. These findings suggest that Coker319ls-1 leaf coloration may be sensitive to cold stress. However, the yellowed plants recovered quickly, with no delayed growth. Normally growing SAS lines were transferred to an experimental field for an examination of their lateral shoot development. Again, the primary lateral shoots were not significantly suppressed in the SAS- $l s$, SAS-bl1, and SAS-rev plants (Table 4), whereas the number and weight of secondary lateral shoots decreased. Furthermore, tertiary lateral shoot formation was nearly completely suppressed (Fig. 2l, $\mathrm{m}$, and $\mathrm{n}$ ). The phenotypes of the RNAi lines in the greenhouse were consistent with the phenotypes of 

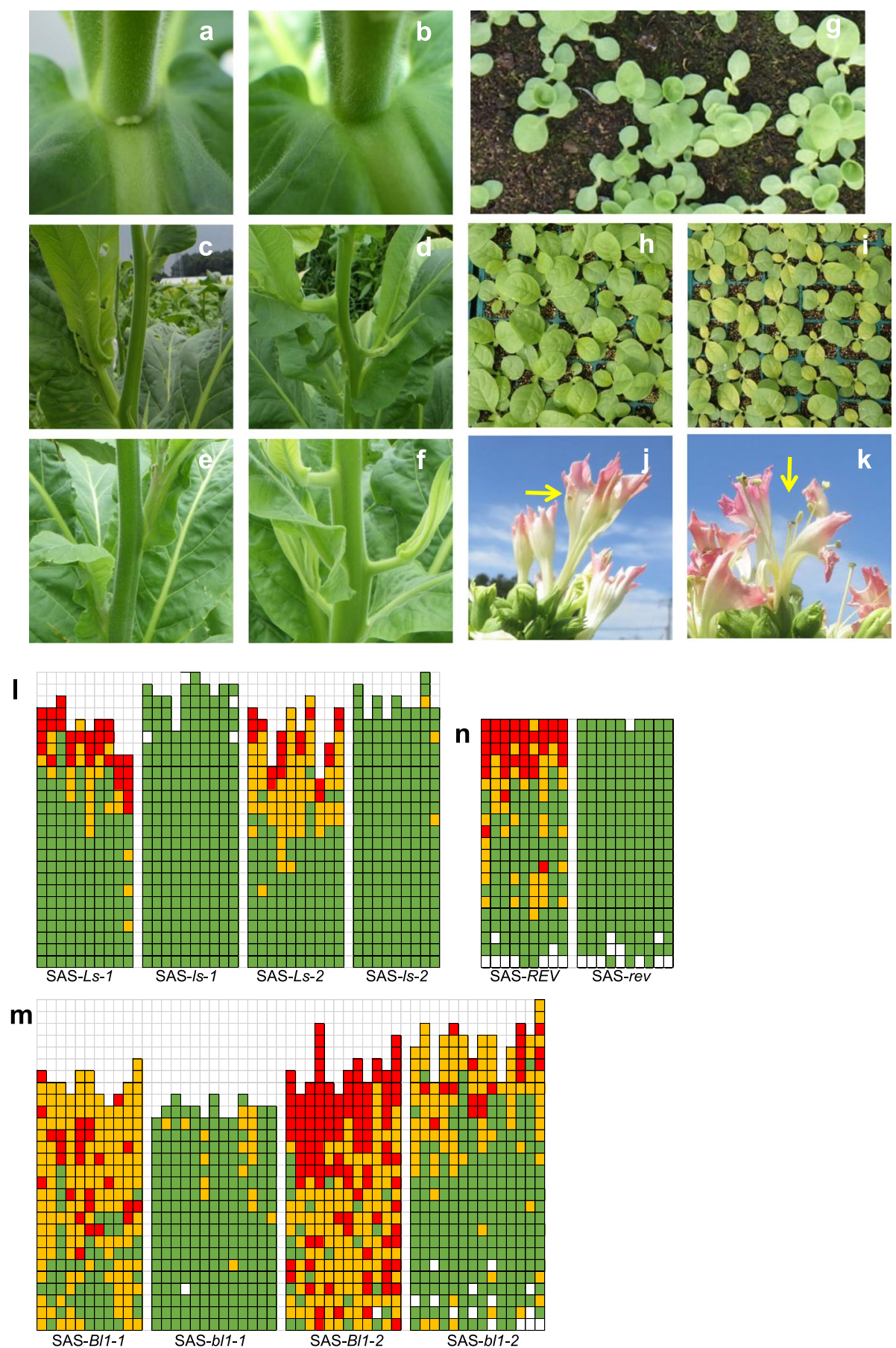

Fig. 2 a, b Adaxial side of the primary lateral shoot base in a wild-type plant (a) and in an RNAi NtLs knock-down plant (b); c-f Primary lateral shoots of SAS-Ls-1 (c), SAS-BI-1-1 (e), SAS-Is-1 (d), and SAS-b/1-1 (f) plants; g-i Seedlings of the SAS-rev mutant (g), 'Coker319' (h), and Coker319-Is-1 (i); j, k Flowers of Coker319-Is-1 plants; I-n Diagrammatic representation of primary, secondary, and tertiary lateral shoot formation in the leaf axils of SAS-Ls and Is lines (I), SAS-B/1 and b/1 lines $(\mathbf{m})$, and SAS-REV and rev lines (n). Each column in (I-n) represents a single plant, with each square representing an individual leaf axil. From bottom to top, the squares represent the leaf positions of plants after topping. Red indicates the presence of primary to tertiary (or more) lateral shoots; yellow indicates the presence of primary to secondary lateral shoots; green indicates the presence of only the primary lateral shoot; and white indicates a lack of lateral shoots 
(a) NtLs

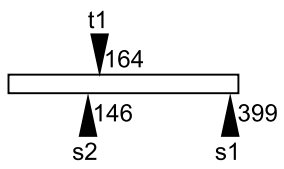

(c) NtREV (b) NtBII

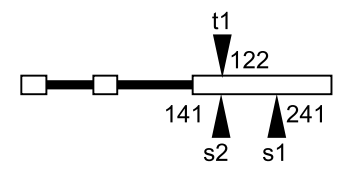

$0.5 \mathrm{~kb}$

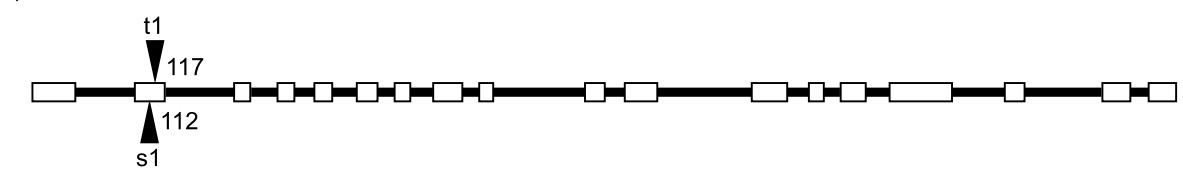

Fig. 3 Locations of chemically induced loss-of-function mutations. Numbers indicate the positions of the nonsense codons from the start of the open reading frame in $\mathbf{a} N t L s, \mathbf{b} N t B / 1$ and $\mathbf{c} N t R E V$. Exons and introns are represented by open boxes and black lines, respectively

the EMS mutants in the field. Interestingly, the position of the primary lateral shoots shifted upward in the SAS- $l s$ and SAS-bl1 mutant lines (Fig. $2 \mathrm{~d}$ and f) in the field and greenhouse (data not shown). A similar shift was not observed in the RNAi lines grown in the greenhouse (Fig. 2b). The positions of the secondary and tertiary lateral shoots in the mutant lines and the RNAi lines were unchanged. Only the position of the primary lateral shoots in the mutant lines of $N t L s$ and $N t B l 1$ was affected, but the reasons for this shift remain unclear.

\section{Field trials in commercial production areas}

Field trials in regions used for the commercial production of tobacco were conducted to examine the agronomic characteristics of the mutant lines. The $l s-1$ and bl1-1 mutations were introduced into a flue-cured tobacco variety 'Coker319' widely cultivated in Japan (Nicotiana tabacum L. cv. Coker319) via backcross breeding (Table 3). Because of space limitations, the rev mutation was not tested.
The results of the analyses of Coker319-ls-1 at four locations and Coker319-bl1-1 at two locations are presented in Table 5. A plot comprising 10 plants was replicated twice for each line at all locations. Overall, the mutant lines did not differ much from the original 'Coker319' plants in terms of leaf yield or other parameters, although small, but statistically significant, differences were detected. Specifically, compared with 'Coker319' plants, the Coker319-ls-1 plants at one or more locations were taller, had larger (length and width) and darker leaves, and flowered later. In contrast, the Coker319-bl1-1 plants at one or two locations were shorter and produced more leaves, which were smaller and less intensely colored (at $1 / 2$ from the top), than the 'Coker319' plants. Therefore, the mutant lines were appropriate for the subsequent trials. Additionally, the development of lateral shoots, especially the secondary and tertiary lateral shoots, was inhibited. A preliminary survey revealed that the labor necessary for removing lateral shoots was $52 \%$ lower for Coker319- $l s-1$ plants than for 'Coker319' plants (Supplemental Table S3).

Table 3 Mutant lines and wild-type segregants characterized in field trials

\begin{tabular}{|c|c|c|c|}
\hline Line code & Target gene & Genotype & Generation \\
\hline SAS-IS-1 & NtLs & sts $1 / t 1 t 1$ & $(\mathrm{M} 2 \times \mathrm{M} 2) \mathrm{F} 3$ \\
\hline SAS-LS-1 & NtLs & SS/TT & $(\mathrm{M} 2 \times \mathrm{M} 2) \mathrm{F} 3$, wild type segregant \\
\hline Coker319-/s-1 & NtLs & s1s1/t1t1 & $(($ Coker319 $\times(\mathrm{M} 2 \times \mathrm{M} 2) \mathrm{F} 1) \times$ Coker319) BC6F3 \\
\hline SAS-Is-2 & NtLs & $s 2 s 2 / t 1 t 1$ & $(\mathrm{M} 2 \times \mathrm{M} 2) \mathrm{F} 3$ \\
\hline SAS-LS-2 & NtLs & SS/TT & $(\mathrm{M} 2 \times \mathrm{M} 2) \mathrm{F} 3$, wild type segregant \\
\hline SAS-b/1-1 & $N+B / 1$ & $s 2 s 2 / t 1 t 1$ & $(\mathrm{M} 2 \times \mathrm{M} 2) \mathrm{F} 3$ \\
\hline SAS-B/1-1 & $N+B / 1$ & SS/TT & $(\mathrm{M} 2 \times \mathrm{M} 2) \mathrm{F} 3$, wild type segregant \\
\hline Coker319-b/1-1 & $N+B / 1$ & $s 2 s 2 / t 1 t 1$ & $(($ Coker319 $\times(\mathrm{M} 2 \times \mathrm{M} 2) \mathrm{F} 1) \times$ Coker319) BC6F3 \\
\hline SAS-b/1-2 & $N t B / 1$ & s1s1titt & $(\mathrm{M} 2 \times \mathrm{M} 2) \mathrm{F} 3$ \\
\hline SAS-B/1-2 & $N+B / 1$ & SS/TT & $(\mathrm{M} 2 \times \mathrm{M} 2) \mathrm{F} 3$, wild type segregant \\
\hline SAS-rev & NtREV & s1s $1 / t 1 t 1$ & $(\mathrm{M} 2 \times \mathrm{M} 2) \mathrm{F} 3$ \\
\hline SAS-REV & NtREV & $S S / T T$ & $(\mathrm{M} 2 \times M 2) \mathrm{F} 3$, wild type segregant \\
\hline
\end{tabular}


Table 4 Lateral shoot production in field trials at an experimental site

\begin{tabular}{|c|c|c|c|c|c|c|c|}
\hline \multirow[t]{2}{*}{ Trial No } & \multirow[t]{2}{*}{ Line code } & \multicolumn{2}{|c|}{ Primary lateral shoots } & \multicolumn{2}{|c|}{ Secondary lateral shoots } & \multicolumn{2}{|c|}{ Tertiary lateral shoots } \\
\hline & & Number \pm SD & Weight $(\mathbf{g}) \pm S D$ & Number \pm SD & Weight $(\mathbf{g}) \pm$ SD & Number \pm SD & Weight $(\mathbf{g}) \pm$ SD \\
\hline \multirow[t]{4}{*}{1} & SAS-LS-1 & $20.60 \pm 1.65$ & $36.79 \pm 15.34$ & $5.60 \pm 1.78$ & $9.99 \pm 5.09$ & $3.10 \pm 0.99$ & $4.27 \pm 2.46$ \\
\hline & SAS-Is-1 & $23.10 \pm 1.37$ & $18.42 \pm 7.09$ & $0.00 \pm 0.00^{\mathrm{a}}$ & $0.00 \pm 0.00^{\mathrm{a}}$ & $0.00 \pm 0.00^{\mathrm{a}}$ & $0.00 \pm 0.00^{\mathrm{a}}$ \\
\hline & SAS-LS-2 & $20.20 \pm 2.30$ & $37.44 \pm 18.29$ & $8.40 \pm 2.55$ & $22.33 \pm 9.33$ & $1.90 \pm 1.10$ & $3.71 \pm 3.21$ \\
\hline & SAS-Is-2 & $22.56 \pm 1.33$ & $32.03 \pm 11.72$ & $0.33 \pm 0.71^{\mathrm{a}}$ & $0.50 \pm 1.10^{\mathrm{a}}$ & $0.00 \pm 0.00^{\mathrm{a}}$ & $0.00 \pm 0.00^{\mathrm{a}}$ \\
\hline \multirow[t]{4}{*}{2} & SAS-BI1-1 & $21.10 \pm 1.22$ & $57.38 \pm N C$ & $16.00 \pm 3.61$ & $64.98 \pm N C$ & $2.30 \pm 1.75$ & $17.67 \pm N C$ \\
\hline & SAS-b/1-1 & $18.80 \pm 0.80$ & $42.87 \pm N C$ & $1.46 \pm 2.18^{\mathrm{a}}$ & $8.67 \pm N C$ & $0.00 \pm 0.00^{\mathrm{a}}$ & $0.00 \pm N C$ \\
\hline & SAS-B/1-2 & $22.00 \pm 1.71$ & $155.01 \pm N C$ & $19.83 \pm 2.86$ & $56.13 \pm N C$ & $12.17 \pm 5.86$ & $57.02 \pm N C$ \\
\hline & SAS-b/1-2 & $23.60 \pm 2.28$ & $95.94 \pm N C$ & $8.36 \pm 3.82^{\mathrm{a}}$ & $49.57 \pm N C$ & $1.43 \pm 1.70^{\mathrm{a}}$ & $5.38 \pm N C$ \\
\hline \multirow[t]{2}{*}{3} & SAS-REV & $19.89 \pm 0.60$ & $85.11 \pm 39.78$ & $8.78 \pm 2.91$ & $35.63 \pm 12.48$ & $5.33 \pm 2.96$ & $17.10 \pm 14.11$ \\
\hline & SAS-rev & $19.80 \pm 0.63$ & $78.42 \pm 29.98$ & $0.00 \pm 0.00^{\mathrm{a}}$ & $0.00 \pm 0.00^{\mathrm{a}}$ & $0.00 \pm 0.00^{\mathrm{a}}$ & $0.00 \pm 0.00^{\mathrm{a}}$ \\
\hline
\end{tabular}

NC Not calculable

a Significantly different from the wild-type line(s) in the same trial at the $1 \%$ level as determined by the $t$-test

\section{Flower development and fertility}

In the greenhouse and field, the petals of most of the flowers of SAS-ls-1, SAS-ls-2, and Coker319-ls-1 plants were split (Fig. 2j). In severe cases, pistils and stamens were completely exposed (Fig. 2k). As compared to naturally pollinated WT flowers, the seed yield from the abnormal flowers of Coker319-ls-1 was decreased by $35 \%$ upon natural pollination and 55\% upon hand pollination (Supplemental Fig. S1).

The Coker319-bl1-1 plants produced 73\% fewer flowers than the wild-type plants in the field trials (Supplemental Fig. S2). The seed yield per flower of Coker319-bl1-1 was $28 \%$ less than Coker319 (Supplemental Fig. S1).

\section{Discussion}

Genetic inhibition of lateral shoot formation was attempted in tobacco. Two groups of tobacco genes were selected to examine the effects of RNAi on lateral shoot formation. One set of candidate genes comprised homologs of the genes reportedly involved in the formation of lateral shoots in other plants. Such genes have been studied most extensively in Arabidopsis. The following eight genes or gene families were identified: $L A S$ [2], RAX family [6, 7], ROX [10], REV [11, 12], CUC family [13-15], LOF [16], LOM [19], and FHY3 [20]. A BLAST search identified tobacco homologs for these genes, with the exception of $R O X$, which was reported after the BLAST search was performed. The VE7 gene, which is homologous to $R O X$, was included in another gene set consisting of genes revealed to be highly expressed in the primordial stages of tobacco axillary buds by an RNA-seq analysis. A homolog of CUC3, $V E 12$, was also in the second gene set. Therefore, tobacco and Arabidopsis apparently share similar sets of genes.
Transgenic lines in which NtLs, NtBl1, NtREV, VE7, or $V E 12$ expression was knocked down by RNAi as well as lines with chemically induced mutations to NtLs, NtBl1, or NtREV exhibited inhibited secondary lateral shoot formation. The NtLs, NtBl1, NtREV, VE7, and VE12 genes are respectively homologs of $L A S, R A X 1, R E V, R O X$, and $C U C 3$. Their reported expression patterns are consistent with the RNA-seq data presented herein. Additionally, mutations in $L A S, L s$ (in tomato), and NtLs affect flower morphology. The stamens of las mutant plants are abnormally short [2]. Other studies proved that petal development is suppressed in $l s$ mutants and that the petals are mostly smaller than the wild-type petals $[1,29]$. Moreover, decreased pollen production and seed production were commonly observed. Mutations in Blind [5], which is a tomato ortholog of RAX1, and NtBl1 result in a decrease in the number of flowers. Therefore, $N t L s, N t B l 1, N t R E V, V E 7$, and VE12 might be considered respectively as the orthologs of $L A S, R A X 1, R E V, R O X$, and $C U C 3$.

Some of the observed characteristics of the knock-down and chemically induced mutant tobacco plants were not reported for other plants. Tobacco primary lateral shoot formation was not suppressed, in contrast to the inhibited development of secondary and tertiary lateral shoots. Furthermore, the positions of the primary lateral shoots shifted upward in the tobacco mutants of NtLs and NtBl1. The mechanisms underlying the differences between plant species remain unclear; however, these differences are not surprising because the diversity in the regulation of lateral branching is a key factor associated with the considerable variety of plant shapes. 


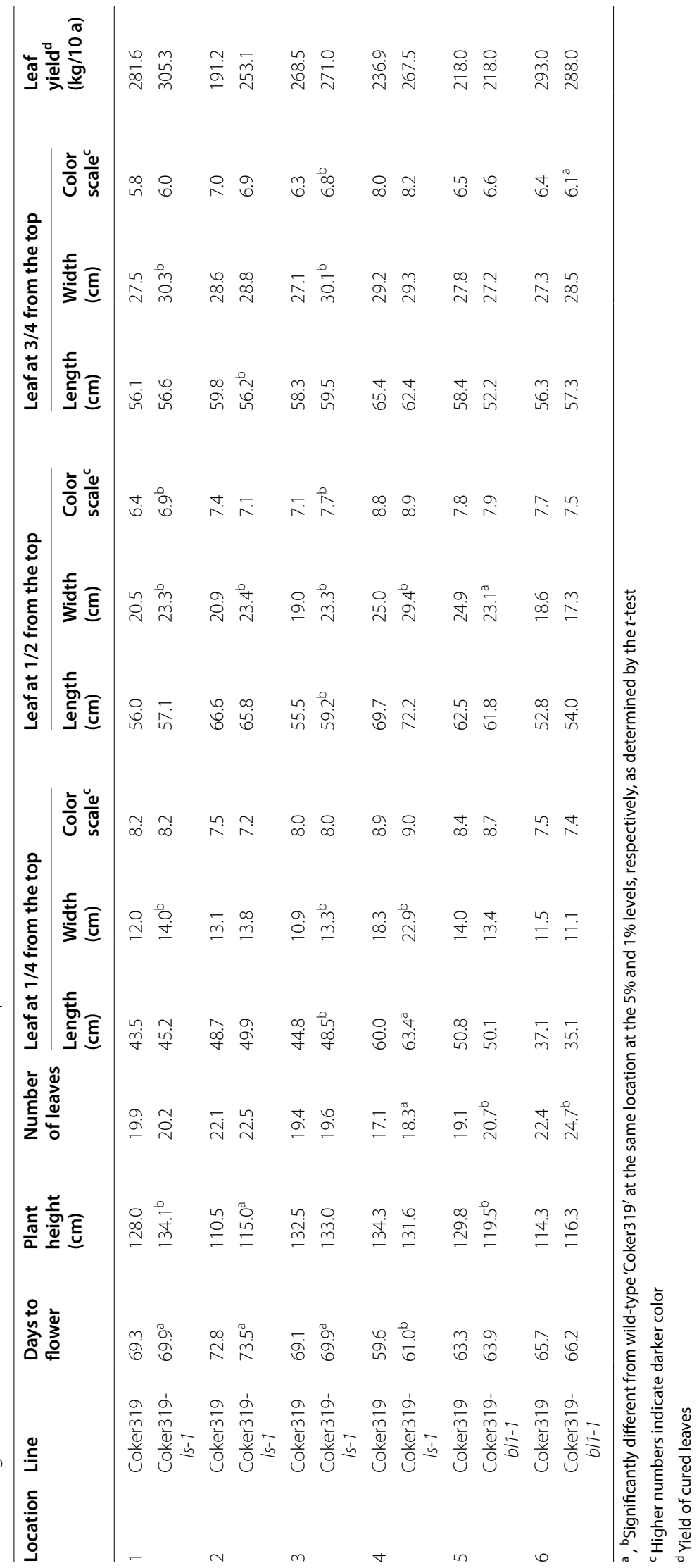


The other analyzed tobacco homologs were ineffective in the knock-down study. The expression of these genes was either undetectable or was not specific to the axillary bud primordial stages. They were probably nonfunctional homologs of genes in other plant species.

The candidate genes in the second set were highly expressed in the axillary bud primordial stages. Twentyfour genes were selected on the basis of the RNA-seq analysis. They were highly and specifically expressed in the EA or VE tissues. However, with the exception of $V E 7$ and $V E 12$, knocking down the expression of these genes did not significantly inhibit lateral shoot formation. Accordingly, these genes might not have crucial regulatory roles related to lateral shoot development. Alternatively, their functions might be redundant in tobacco.

Two mutant tobacco lines with the 'Coker319' genetic background were examined in field trials at commercial tobacco production sites. These lines were satisfactory for tobacco leaf production and the trials should be repeated. Regarding the consequences of the inhibition of secondary and tertiary lateral shoot formation, the labor required for removing lateral shoots was assessed in a preliminary survey. Although cold sensitivity at the seedling stage and some floral abnormalities that decreased seed yield were observed in the NtLs-mutated plants, these characteristics are unlikely to affect tobacco leaf production practices.

The 'Coker319' derivatives examined in this study are potentially useful new tobacco varieties. They may decrease the labor and time required by tobacco growers to control secondary and tertiary lateral shoot formation. Additionally, breeding other varieties with mutations in $N t L s$ and/or NtBl1 and further characterizing the NtLs, $N t B l 1, N t R E V, V E 7$, and $V E 12$ genes are among the important objectives that should be pursued in future studies.

\section{Conclusions}

In this study, we attempted to develop tobacco lines with inhibited lateral shoot formation. There is a considerable demand for such lines among tobacco farmers. The RNA interference of five genes and the chemically induced mutations to three genes significantly suppressed lateral shoot development in tobacco under greenhouse and field conditions. Two mutant lines were evaluated regarding their agronomic performance in field trials conducted at commercial production sites. Although primary lateral shoot formation was not inhibited in the examined lines, the decreased production of secondary and tertiary lateral shoots may have implications for improved commercial tobacco production.

\section{Methods}

\section{Plant materials}

Nicotiana tabacum L. cv. Petit Havana SR-1 was provided by Fukui Prefectural University in 1995 and used for RNA sequencing and RNA interference. Nicotiana tabacum L. cv. Tsukuba 1 was developed at Japan Tobacco Inc. in 1980 and used for cloning of genes and development for chemically induced mutants. Nicotiana tabacum L. cv. Coker319 was provided by Coker's Pedigreed Seed Co. in 1963 and used for breeding.

\section{BLAST analysis}

The genes used as queries for BLAST searches of the NCBI BLAST database (http://blast.ncbi.nlm.nih.gov/ Blast.cgi) and a database comprising tobacco cDNA sequences accumulated by a number of in-house studies are listed in Table 1.

\section{RNA sequencing}

Apical tissues of shoots were collected from 'Petit Havana SR-1' tobacco seedlings at 29-37 days after germination, after which they were fixed in an ice-cold 3:1 solution of ethanol and acetic acid. The protocols used to prepare paraffin-embedded sections and laser-microdissection were previously described by Takahashi et al. [30]. Total RNA extracted using the PicoPure ${ }^{\mathrm{TM}}$ RNA isolation Kit (Thermo Fisher Scientific Inc.) was examined using the Agilent 2100 Bioanalyzer and the RNA 6000 Pico kit (Agilent Technologies Inc.). Next, cDNA was synthesized using an oligo-dT primer with the T7 promoter sequence, after which antisense RNA was transcribed using T7 RNA polymerase. The antisense RNA was served as the template for synthesizing cDNA using random primers. After a fragmentation step, the cDNA (400-1,000 bp) containing the $3^{\prime}$ end was collected and the $5^{\prime}$ end of the fragments were sequenced using the 454 GS FLX system. Files containing these sequences have been deposited at DDBJ (DRA011900). The obtained reads were assembled using Newbler (version 2.6).

\section{Gene cloning}

After extracting coding sequence information from the GenBank and in-house cDNA databases, 5'- or 3'-RACE was performed using the SMARTer RACE cDNA Amplification Kit (Clontech) to obtain full-length cDNA clones of the coding sequences. Total RNA was extracted from dormant axillary buds, shoot apices, flower buds, or roots (Magtration ${ }^{\circledR}$; Precision System Science Co., Ltd.). Amplified fragments were sequenced using the BigDye ${ }^{\circledR}$ (version 3.1) Cycle Sequencing Kit (Thermo Fisher Scientific Inc.) and the 3730xl DNA Analyzer (Thermo Fisher Scientific Inc.). Standard procedures were used for DNA manipulations [31]. Information regarding the obtained sequences is provided in Supplemental Table S4. 


\section{RNA interference}

The primers listed in Supplemental Table S2 were used to amplify trigger sequences from the cloned cDNA or RNA isolated from dormant axillary buds, shoot apices, flower buds, or roots collected from 'Petit Havana SR-1' tobacco plants. The amplified fragments were cloned into the $\mathrm{pENTR}^{\mathrm{TM}} / \mathrm{D}$-TOPO vector (Takara Bio) and then introduced into a modified pHellsgate12 binary vector [32] containing an expression cassette comprising a gene encoding the green fluorescent protein under the control of the CaMV 35S promoter. The binary vectors were inserted into Agrobacterium tumefaciens strain LBA4404 cells, which were then used to transform 'Petit Havana SR-1' tobacco plants. The transgene locus number was determined on the basis of the segregation ratios for the green fluorescence in the $T_{1}$ generation. Target gene expression levels were analyzed by quantitative PCR. Briefly, RNA extracted from the leaves, roots, or aboveground tissues excluding leaves was used as the template for synthesizing cDNA using the PrimeScript RT reagent kit with gDNA Eraser (Takara Bio). The quantitative PCR analysis was performed using the TaqMan Fast Advanced Master Mix (Thermo Fisher Scientific Inc.) and the StepOnePlus system (Thermo Fisher Scientific Inc.). Details regarding the primers and probes are listed in Supplemental Table S5. The gene encoding elongation factor- $1 \alpha$ (AF120093) was used as the internal reference control. The relative expression of the target genes in the RNAi lines was compared with the expression in null segregant controls lacking the transgene.

\section{Chemically induced mutants}

A library for Nicotiana tabacum L. cv. Tsukuba 1 mutated with EMS by Tajima et al. [33] was screened as described by Takakura et al. [34] using the primers listed in Supplemental Table S6.

\section{Plant cultivation}

Tobacco plants were grown in pots $(12 \mathrm{~cm})$ containing soil in a greenhouse at $25^{\circ} \mathrm{C}$ under natural day-length conditions or in a growth chamber with a 12-h light $\left(25^{\circ} \mathrm{C}\right): 12$-h dark $\left(18{ }^{\circ} \mathrm{C}\right)$ cycle. They were also grown in an experimental field at the Leaf Tobacco Research Center of Japan Tobacco Inc. or in fields used for commercial tobacco production in southwestern Japan. The fields were managed according to the respective standard practices. When flower buds emerged, the plants were topped off, after which the lateral shoots were removed and measured weekly for 5 weeks in the growth chamber or for 8 weeks in the field. Leaf color was scored using the Leaf Color Chart 2019A for tobacco (Fujihira Industry; Supplemental Table S7).

\section{Abbreviations}

LS, LAS: Lateral suppressor; MOC1: Monoculm 1; RAX: Regulator of axillary meristems; MYB: Myeloblastosis; LAX: Lax panicle; BA1: Barren stalk 1; bHLH: Basic helix-loop-helix; ROX: Regulator of axillary meristem formation; REV: Revoluta; CUC: Cup-shaped cotyledon; LOF: Lateral organ fusion; HAM: Hairy meristem; LOM: Lost meristem; FHY3: Far-red elongated hypocotyl3; RNAi: RNA interference; NCBI: National Center for Biotechnology Information; BLAST: Basic local alignment search tool; Bl: Blind; RNA-seq: RNA sequencing; SAM: Shoot apical meristem; NAC: Petunia NAM and Arabidopsis ATAF1, ATAF2, and CUC2; EMS: Ethyl methanesulfonate; RACE: Rapid amplification of cDNA ends; CaMV: Cauliflower mosaic virus; SD: Standard deviation.

\section{Supplementary Information}

The online version contains supplementary material available at https://doi. org/10.1186/s12870-021-03008-3.

Additional file 1: Supplemental Table S1. Expression of genes in several tissues and organs. Supplemental Table S2. Primers for amplifying trigger DNA. Supplemental Table S3. Labor hours for manually removing lateral shoots in field trials at commercial production sites. Supplemental Table S4. Accession numbers of genes screened by RNA-seq. Supplemental Table S5. Primers and probes for quantitative PCR. Supplemental Table S6. Primers for screening mutations. Supplemental Table S7. CIELAB color scores (International Commission on Illumination; http://cie. co.at/) determined using Leaf Color Chart 2019A for tobacco.

Additional file 2: Supplemental Fig. S1. Seed yield of mutant lines. Statistical significance was determined using the $t$-test $\left({ }^{* *} p<0.01\right)$. NS: not significant.

Additional file 3: Supplemental Fig. S2. Number of flowers in Coker319b/1-1 plants. Statistical significance was determined using the $t$-test $\left({ }^{* *} p<0.01\right)$.

\section{Acknowledgments}

The authors acknowledge Dr. Mikio Nakazono and Dr. Hirokazu Takahashi for their technical support during the laser micro-dissection and RNA extraction. We also acknowledge Dr Toshihiko Komari for his kind, critical, and careful reading of the manuscript. We would like to express our appreciation for Dr. Satoko Oguro, Ayako Koizumi, Dr. Mai Tsukahara, Nanae Sato, Sayaka Koike, and Akiko Takahashi for their excellent technical assistance.

\section{Authors' contributions}

$\mathrm{KH}, \mathrm{SS} 1, \mathrm{TN} 1$, and SS2 designed the research and experiments. $\mathrm{KH}, \mathrm{SS} 1, \mathrm{MA}, \mathrm{YN}$, TN1, TK, TN2, and SS2 performed the experiments. KH, SS1, and SS2 regularly discussed the research progress and developed the research strategy. $\mathrm{KH}$ and SS2 wrote the manuscript. SS1 and SS2 correspond to Seiki Sato and Shoichi Suzuki, respectively. TN1 and TN2 correspond to Takashi Nakamura and Tsuyoshi Naragino, respectively. All authors have read and approved the manuscript.

\section{Funding}

Japan Tobacco Inc., with which all authors are affiliated, is the sole funder of the research. This study was performed as one of the activities at department of research and development at Tobacco Business division.

\section{Availability of data and materials}

All data generated or analyzed during this study are included in the manuscript and its supplementary files. All images depicted in the figure are our own. The sequence information of tomato, Arabidopsis and tobacco genes is available from Solanaceae Genomics Network (https://solgenomics.net/), GenBank (https://www.ncbi.n/m.nih.gov/genbank/) and DNA DataBank of Japan (https://www.ddbj.nig.ac.jp/index-e.html). The raw reads of next-generation sequencing-based obtained in this study is available from the DDBJ/EMBL/ NCBI under the accession number DRA011900 (https://ddbj.nig.ac.jp/DRASe arch/submission?acc=DRA011900). The datasets generated or analyzed during the current study are available from the corresponding author for noncommercial purposes. 


\section{Declarations}

Ethics approval and consent to participate

Not applicable.

\section{Consent for publication}

Not applicable.

\section{Competing interests}

Japan Tobacco Inc. funded production of all the research and hold a patent regarding RNAi tobaccos and chemically induced tobacco mutants. Patent WO/2017/170796A1, Japan Tobacco Inc., Tobacco plant body and method for producing the same.The authors declare that they have no competing interests. All authors are affiliated with Japan Tobacco Inc., a funder of this study.

Received: 21 December 2020 Accepted: 5 May 2021

Published online: 27 May 2021

\section{References}

1. Schumacher K, Schmitt T, Rossberg M, Schmitz G, Theres K. The Lateral suppressor (Ls) gene of tomato encodes a new member of the $\mathrm{VHIID}$ protein family. Proc Natl Acad Sci U S A. 1999:96:290-5.

2. Greb T, Clarenz O, Schafer E, Muller D, Herrero R, Schmitz G, Theres K. Molecular analysis of the LATERAL SUPPRESSOR gene in Arabidopsis reveals a conserved control mechanism for axillary meristem formation. Genes Dev. 2003:17:1175-87.

3. Li X, Qian Q, Fu Z, Wang Y, Xiong G, Zeng D, Wang X, Liu X, Teng S, Hiroshi F, et al. Control of tillering in rice. Nature. 2003;422:618-21.

4. Mapelli S, Kinet JM. Plant growth regulator and graft control of axillary bud formation and development in the TO-2 mutant tomato. Plant Growth Regul. 1992;11:385-90.

5. Schmitz G, Tillmann E, Carriero F, Fiore C, Cellini F, Theres K. The tomato Blind gene encodes a MYB transcription factor that controls the formation of lateral meristems. Proc Natl Acad Sci U S A. 2002;99:1064-9.

6. Keller T, Abbott J, Moritz T, Doerner P. Arabidopsis REGULATOR OF AXILLARY MERISTEMS1 controls a leaf axil stem cell niche and modulates vegetative development. Plant Cell. 2006;18:598-611.

7. Müller D, Schmitz G, Theres K. Blind homologous R2R3 Myb genes control the pattern of lateral meristem initiation in Arabidopsis. Plant Cell. 2006;18:586-97.

8. Komatsu K, Maekawa M, Ujiie S, Satake Y, Furutani I, Okamoto H, Shimamoto K, Kyozuka J. LAX and SPA: major regulators of shoot branching in rice. Proc Natl Acad Sci U S A. 2003;100:11765-70.

9. Gallavotti A, Zhao Q, Kyozuka J, Meeley RB, Ritter MK, Doebley JF, Pè ME, Schmidt RJ. The role of barren stalk1 in the architecture of maize. Nature. 2004;432:630-5.

10. Yang F, Wang Q, Schmitz G, Müller D, Theres K. The bHLH protein ROX acts in concert with RAX1 and LAS to modulate axillary meristem formation in Arabidopsis. Plant J. 2012;71:61-70.

11. Talbert PB, Adler HT, Parks DW, Comai L. The REVOLUTA gene is necessary for apical meristem development and for limiting cell divisions in the leaves and stems of Arabidopsis thaliana. Development. 1995;121:2723-35.

12. Otsuga D, DeGuzman B, Prigge MJ, Drews GN, Clark SE. REVOLUTA regulates meristem initiation at lateral positions. Plant J. 2001;25:223-36.

13. Aida M, Ishida T, Fukaki H, Fujisawa H, Tasaka M. Genes involved in organ separation in Arabidopsis: an analysis of the cup-shaped cotyledon mutant. Plant Cell. 1997:9:841-57.

14. Aida M, Ishida T, Tasaka M. Shoot apical meristem and cotyledon formation during Arabidopsis embryogenesis: interaction among the CUPSHAPED COTYLEDON and SHOOT MERISTEMLESS genes. Development. 1999:126:1563-70.
15. Takada S, Hibara K, Ishida T, Tasaka M. The CUP-SHAPED COTYLEDON1 gene of Arabidopsis regulates shoot apical meristem formation. Development. 2001;128:1127-35.

16. Lee DK, Geisler M, Springer PS. LATERAL ORGAN FUSION1 and LATERAL ORGAN FUSION2 function in lateral organ separation and axillary meristem formation in Arabidopsis. Development. 2009;136:2423-32.

17. Stuurman J, Jäggi F, Kuhlemeier C. Shoot meristem maintenance is controlled by a GRAS-gene mediated signal from differentiating cells. Genes Dev. 2002:16:2213-8.

18. David-Schwartz R, Borovsky Y, Zemach H, Paran I. CaHAM is autoregulated and regulates CaSTM expression and is required for shoot apical meristem organization in pepper. Plant Sci. 2013;203-204:8-16.

19. Schulze S, Schäfer BN, Parizotto EA, Voinnet O, Theres K. LOST MERISTEMS genes regulate cell differentiation of central zone descendants in Arabidopsis shoot meristems. Plant J. 2010;64:668-78.

20. Stirnberg P, Zhao S, Williamson L, Ward S, Leyser O. FHY3 promotes shoot branching and stress tolerance in Arabidopsis in an AXR1-dependent manner. Plant J. 2012;71:907-20.

21. Wang W, Tai S, Wang L, Liu G-s, Liu F-x, Gao X-m, Sun Y-h. Construction of RNAi vector of NtLS gene and its transformation in tobacco. Chin Tob Sci. 2011;32:31-5.

22. Riechers DE, Timko MP. Structure and expression of the gene family encoding putrescine $\mathrm{N}$-methyltransferase in Nicotiana tabacum: new clues to the evolutionary origin of cultivated tobacco. Plant Mol Biol. 1999:41:387-401.

23. Murad L, Lim KY, Christopodulou V, Matyasek R, Lichtenstein CP, Kovarik A Leitch AR. The origin of tobacco's T genome is traced to a particular lineage within Nicotiana tomentosiformis (Solanaceae). Am J Bot. 2002:89:921-8.

24. Gavilano LB, Coleman NP, Bowen SW, Siminszky B. Functional analysis of nicotine demethylase genes reveals insights into the evolution of modern tobacco. J Biol Chem. 2007;282:249-56.

25. Hibara K, Karim MR, Takada S, Taoka K, Furutani M, Aida M, Tasaka M. Arabidopsis CUP-SHAPED COTYLEDON3 regulates postembryonic shoot meristem and organ boundary formation. Plant Cell. 2006;18:2946-57.

26. Parrish S, Fleenor J, Xu S, Mello C, Fire A. Functional anatomy of a dsRNA trigger: differential requirement for the two trigger strands in RNA interference. Mol Cell. 2000:6:1077-87.

27. Xu P, Zhang Y, Kang L, Roossinck MJ, Mysore KS. Computational estimation and experimental verification of off-target silencing during posttranscriptional gene silencing in plants. Plant Physiol. 2006;142:429-40.

28. Rushton PJ, Bokowiec MT, Han S, Zhang H, Brannock JF, Chen X, Laudeman TW, Timko MP. Tobacco transcription factors: novel insights into transcriptional regulation in the Solanaceae. Plant Physiol. 2008;147:280-95.

29. Groot SPC, Keizer LCP, de Ruiter W, Dons JJM. Seed and fruit set of the lateral suppressor mutant of tomato. Sci Hortic. 1994;59:157-62.

30. Takahashi H, Kamakura H, Sato Y, Shiono K, Abiko T, Tsutsumi N, Nagamura Y, Nishizawa NK, Nakazono M. A method for obtaining high quality RNA from paraffin sections of plant tissues by laser microdissection. J Plant Res. 2010;123:807-13.

31. Sambrook JF, Russell DW. Molecular cloning: a laboratory manual (3-volume set). Cold Spring Harbor: Cold Spring Harbor Laboratory Press; 2001

32. Wesley SV, et al. Construct design for efficient, effective and high-throughput gene silencing in plants. Plant J. 2001;27:581-90.

33. Tajima T, Sato S, Hiyoshi T. Construction of mutant panel in Nicotiana tabacum L. Ann Phytopathol Soc Jpn. 2011;77:258.

34. Takakura Y, Udagawa H, Shinjo A, Koga K. Mutation of a Nicotiana tabacum L. eukaryotic translation-initiation factor gene reduces susceptibility to a resistance-breaking strain of Potato virus Y. Mol Plant Pathol. 2018;19:2124-33.

\section{Publisher's Note}

Springer Nature remains neutral with regard to jurisdictional claims in published maps and institutional affiliations. 